\title{
Otto Sundt
}

\section{»Fordismus in Spanien \\ Eine Entgegnung auf Volker Wellhöners Interpretation}

In PROKLA Nr. 80 erschien ein Aufsatz von Volker Wellhöner, mit dem der Autor den ambitionierten aber wenig originellen Versuch macht, die Prosperitätsphase der spanischen Wirtschaft von 1959 bis 1973 neu zu interpretieren bzw. im Regulations- und Fordismusparadigma zu verorten.

Ausgangspunkt seiner Überlegungen sind die nostalgischen Befindlichkeiten eines Tourismusveteranen, kulinarische Gedankengänge eines Linksintellektuellen, der unterhaltsame Kriminalromane schreibt, und die Erfahrung des Autors, daß in Spanien eine gewisse Vereinheillichung des pharmazeutischen Angebots sich bemerkbar macht. Wellhöner zieht daraus den Schluß, daß Spanien charakteristische Eigenarten verloren habe und »bestenfalls bedingt sinnvolle Untersuchungseinheit« (ebd., S. 156) sein könne. Er schiebt kritische Einwände gegen die Regulationstheorie beiseite und ignoriert konsequent Untersuchungen, die dem von ihm favorisierten theoretisschen Ansatz widersprechen. Die Kritik am Regulationsansatz erfährt durch Wellhöners Aufsatz jedoch eine besondere Berechtigung, weil hier exemplarisch die Unzulänglichkeiten dieses Ansatzes demonstriert werden: 1. Der Regulationsansatz und das Fordismuskonzept sind nicht in der Lage, das kritisierte Gleichgewichtstheorem zu widerlegen, weil sie sich nicht auf der Ebene ökonomischer Theorien bewegen (Cartelier/de Vroey 1988). Entsprechend unscharf wird der von Lipietz (1985, S. 113) entwickelte Krisenbegriff; Krisen können dann nur ex post konstatiert werden. Bei der Analyse der jüngsten Vergangenheit und aktueller Prozesse versagt die Regulationstheorie. 2. Obwohl der Regulationsansatz vorgibt, Gleichgewichtstheorien zu kritisieren, impliziert er selbst ein institutionell determiniertes Gleichgewicht (Hübner/Mahnkopf 1988, S. 81). 3. Innerbetriebliche Regulationsweisen werden ignoriert. Im vorliegenden Fall wird sogar die spezifische nationale Regulation unterkomplex dargestellt.

Wellhöner ordnet die bisherigen Untersuchungen und Darstellungen der ókonomischen Entwicklung Spaniens einem »Kontinuitätsparadigma« unter, welches er mit einem Zitat von B. Lutz zu widerlegen glaubt (ebd., S. 154). Durch seine Anwendung des Fordismuskonzeptes auf Spanien unterstellt er, daß der Industrialisierungsprozeß einer immanenten Entwicklungslogik folgt, die einen Verhaltenskodex bestimmt, dem - in Abstraktion vom historischen Raum - regionale Universalitåt zukommt. Doch aus Wellhöners Ausführungen ergibt sich lediglich, daß die von ihm unterstellte Implantation des Fordismus in Spanien einen Bruch bzw. historischen Wandel darstellen würde. Seine Kritik an klassischen Gleichgewichtstheorem und dessen modernen Adaptionen (cbd., S. 155) beschränkt sich darauf, daß diese nicht das analytische Werkzeug liefern, um historische Periodisierungen vomehmen zu können. Fragt sich nur, wozu ein solches Verfahren 
nützlich ist, wenn mit der Benennung von Perioden oder Epochen der Prozeßcharakter der Geschichte negiert wird.

Wellhöners Affinităt zu Gleichgewichtstheorien ist besonders auffällig dort, wo er behauptet, die einzig denkbare Alternative zur Weltmarktorierung sei eine erfolgreiche Importsubstutionspolitik gewesen (ebd., S. 158), die jedoch an der Durchsetzungsschwäche der staatlichen Industrialisierungspolitik des franquistischen Regimes gescheitert sei. Mit dieser Feststellung blendet Wellhöner den Interessenbezug der Importsubstitutionspolitik aus. Importsubstition steht in erster Linie für das Interesse einheimischer Unternehmen, teure Produkte auf einem protektionierten Markt zu verkaufen. Daran aber scheitert der Versuch, komparative Vorteile im internationalen Handel zur Geltung zu bringen (Olson 1985, S. 222) trotz der erheblichen (in der Regel intern erzwungenen) Lohnkostenvorteile. Einmal abgesehen davon, daß Importsubstition und exessiver Protektionismus vorzugsweise zum Repertoire von diktatorischen Regimes jeglicher Provinienz gehören. Von Olson ist zu lernen, daß Diktaturen keineswegs Stabilität und Gleichgewicht gewährleisten; sie fördern privilegierte Gruppen, deren Sonderinteressen destabilisierend wirken (ebd., S. 219).

Für Spanien hat dies Ochoa Westenenk (1986, S. 51-69) nachgeweisen: Er identifiziert die Gruppe der spanischen Bankiers als das Kollektiv, welches zunächst unter der rigiden Autarkiepolitik eine priviligierte Stellung einnehmen konnte, die aber, nachdem sie die Effizienzgrenze dieser Politik erreicht hatte, Änderungen einleitete. Diese Änderungen hatten in erster Linie den Zweck, Spanien als Binnenmarkt für das eigene Kapital zu erschließen (ebd.,S. 15). Importtätigkeit und Kapitalverkehr wurden nur in dem Maße liberalisiert, wie dies zur angestrebten Binnenmarkterschließung notwendig war (ebd.,S. 219, vgl. auch Mata 1979, S. 119). Angesichts des inferioren Ausrüstungsstandes der spanischen Industrie und mangelnder interner technologischer Innovationskapazitäten erwiesen sich die Importe als so notwendig, daß Ochoa Westenenk von einem importinduzierten Wachstumsmodell spricht (ebd.).

Ähnlich verhielt es sich mit ausländischen Direkt- und Kapitalinvestitionen nach Spanien. Sie bildeten die dritte Săule, mit der externe Defizite gestützt bzw. abgedeckt wurden (Ochoa Westenenk 1986, S. 232ff). Als Direktinvestitionen unterlagen sie der Kontrolle des Regimes. Selbst wenn man die in der Zeit von 1960 bis 1975 in Spanien getătigten ausländischen Direktinvestitionen skepstisch beurteilt, so lassen sich - angesichts der weitgehend fehlenden internen produktiven Investitionen - ihre positiven Effekte doch nicht ignorieren (de la Dehensa 1985, S. 77-82). In seinem interessanten Vergleich des Verflechtungsgrades ausgewählter OECD-Staaten konnte de la Dehesa Spanien nur auf einer unterdurchschnittlichen Position verorten (ebd., S. 84); die spezifische Abhăngigkeitsposition, die Wellhöner konstatiert, war für Spanien in dieser Zeit also nicht gegeben.

Nach übereinstimmender Ansicht verschiedener Autoren handelte es sich bei dem vielzitierten Stabilisierungsplan eher um eine Maßnahme zur Sicherung des autoritären Regimes nicht aber um eine Reaktion auf externe Entwicklungen (Ochoa 
Westenenk 1986, S. 81, Bernecker 1990, S. 303, Tamames 1983, S.606ff); daß er zeitweilig einen Boomeffekt hatte, ist auf die günstige externe Konjunktur zurückzuführen. Die im Stabilisierungsplan skizzierte Politik war im wesentlichen Importsubstitionspolitik und moderater Protektionismus (Serrano Sanz 1988, S. 51); auch weiterhin war die spanische Wirtschaft nicht darauf angewiesen, eine konkurrenzfähige Exportindustrie aufzubauen.

Besonders deutlich wurde dies im Bereich der langlebigen Konsumgüter, speziell bei der PKW-Produktion: Ausgehend von einem extrem niedrigen Niveau war zwar die Produktionssteigerung zwischen 1960 und 1970 enorm. Im internationalen Vergleich erreichte die Produktion mit lediglich 450 Tsd. Einheiten im Jahre 1970 jedoch nur einen sehr niedrigen Stand. Der Anteil der importierten Fahrzeuge an den Neuzulassungen in Spanien sank von 6\% im Jahre 1965 auf 3\% im Jahre 1970, um 1975 schließlich bei 1,6\% zu landen. 1970 besaßen von 1000 Spaniern 65 einen PKW. Dies muß vor dem Hintergrund gesehen werden, daß Spanien seit dem letzten Jahrhundert über eine eigene nationale Automobilproduktion verfügte (Farre Terre 1986 und eigene Berechnung). Im Fordismuskonzept spielen das Automobil als Massenkonsumgut und dessen Produktion eine ganz zentrale Rolle. Die vorliegenden Ergebnisse lassen jedoch m. E. nicht erkennen, inwieweit dieses Konzept in Spanien eine Rolle spielte.

Zentraler Analysegegenstand des Regulationsansatzes ist das "Lohnverhältnis«. Bei seiner Analyse des Lohnverhältnisses in der sogannten Liberalisierungsphase des Franco-Regimes verfährt Wellhöner besonders großzügig, d.h. er verfälscht den Gegenstand: Obwohl selbst der von ihm zitierte Toharia hinsichtlich des Lohnverhältnisses im franquistischen Spanien von einem »Pseudo- Fordismus« spricht, hält Wellhöner am Fordismuskonzept fest - weil für ihn die Tendenz einer parallelen Entwicklung von Produktivität und Löhnen die letztlich entscheidende ist. Dagegen läßt sich zunächst darauf verweisen, daß »Produktivität《 als globaler Indikator problematisch ist. Doch selbst in dieser globalen Verwendung ist die behauptete Tendenz nicht eindeutig (vgl. Ochoa Westenenk 1986, S. 159ff). Lohnstruktur und Produktivität haben sich in den einzelnen Industriebranchen sehr unterschiedlich entwickelt (vgl. Mata 1979, S. 117f). Wie Malo de Molina (1982, S. 95f) zeigen konnte, hat das Fehlen freier Gewerkschaften einerseits die Angleichung der Lohnhőhen zwischen den Branchen verhindert, andererseits reduzierte die flexible Lohnstruktur »spill-over-Effekte«. Zu dieser spezifischen spanischen Lohnstruktur kam es trotz aller dirigistischen Versuche, die Lơhne niedrig zu halten. Denn durch die illegale Tätigkeit der $\mathrm{CCOO}$ und im Einklang mit den Produktionserfordemissen der Unternehmen entwickelte sich unter der dirigistischen Decke ein System von Zulagen, Gratifikationen, Prämien etc. (flecos salariales), das einen höheren Anteil am Effektivlohn hatte als die offiziell ausgehandelten und zustimmungspflichtigen »Tariflöhne (Serrano/ Malo de Molina 1979, S. 231ff). Der Charakter der industriellen Beziehungen und die spezifischen Mechanismen der Lohnfindung, die sich unter dem Franco-Regime entwickelten, haben ein spezifisches Muster relativ rigider und geschlossener betrieblicher Räume 
(Pries 1987) geschaffen, das bis heute seine Wirkung tut. Dies gilt vor allem auch hinsichtlich der Lohnstruktur (ebd., S. 33). Fast überflüssig zu erwähnen, daß von einer staatlichen Sozialpolitik und von angemessenen Transferleistungen in Spanien gar keine Rede sein konnte. Dem angeblichen fordistischen Entwicklungsweg stand in Spanien kein adäquates Modell institutioneller Regulation zur Seite so wie es die Theorie voraussetzt. Im Zeitraum von 1959 - 1975 entstanden Formen einer informellen Regulation, die die Flexibilitätserfordernisse der spanischen Industrie überkompensierte. Die Regulation fand auf betrieblicher Ebene statt.

Deregulation und Flexibilisierung werden von Regulationisten als Elemente post" fordistischer Entwicklung charakterisiert. In der Terminologie der Regulationstheorie bedeutet dies, daß der »semi-periphere Fordismus« im Bestreben industrielle Entwicklung nachzuholen, ein post-fordistisches Lohnverhältnis installiert und somit den zentralen Fordismen um einiges voraus ist. Theoretisch bleibt dann nur noch zu klären, was »Post-Fordismus« eigentlich ist.

Wellhöners Aufsatz liest sich in weiten Teilen wie ein posthumer Vorwurf an Franco, den Penetrationsgeliusten des hegemonial struktierten internationalen »Kapitals« nicht adäquaten Widerstand geleistet zu haben und als Nationalist umgefallen zu sein. Dies mag auch als eine besondere Referenz an spanische »Linksintellektuelle« verstanden werden, die einen spezifischen Antiamerikanismus pflegen. Die Kehrseite dieser Argumentation wäre für die spanische Linken allerdings wenig schmeichelhaft: Sie würden dann nämlich beanspruchen, die konsequenteren Nationalisten zu sein. Eine solche Tendenz ist innerhalb der spanischen Linken und besonders bei baskischen und anderen Nationalismen, die sich auch »links « etikettieren, tatsächlich virulent. Wellhöners spanische Impressionen sind subjektiv und als solche literarisch verwertbar; zur Feststellung, Spanien sei kulturell begradigt worden, taugen sie nicht. Solange die Spanier Anglizismen vermeiden und ihrerseits sprachlich hispanisieren - worin sich mehr als nur typischer spanischer Stolz ausdrückt -, solange in Spanien Stierkämpfe stattfinden etc., muß mit einer kulturellen Begradigung wohl nicht gerechnet werden. Es gibt eine lange Liste von spanischen Eigenarten, die den spanischen Euphemismus »ya somos europeos« Lügen strafen. Spanischer Partikularismus (Lokal- und Regionalpatriotismus) und Nationalismus, der in Europa nicht ein integratives Ziel, sondern die nationale Verwirklichung auf höherer Ebene sieht, ist in Spanien an der Tagesordnung (Frey 1988, S. 154ff)。

Das Zusammentreffen von Stagnation und Krise des Franco-Regimes mit externen Krisentendenzen war eher zufälliger Art. Die unter dem Primat des Machterhalts stehende Wirtschaftspolitik des franquistischen Spaniens hatte strukturkonservierende Effekte, deren Widersprüche durch günstige externe Umstände zeitweilig kaschiert wurden. Letztendlich haben aber strukturelle Insuffizienzen der spanischen Industrie und interne Widersprüche das Liberalisierungsprojekt zum Scheitem gebracht. Ähnlich wie es z.Z. am Beispiel der osteuropäischen Volkswirtschaften zu beobachten ist, brachte der Zusammenbruch des Regimes, ausgelöst 
durch die biologischen Grenzen eines Diktators, diese Widersprüche unmittelbar zum Vorschein. Der von einigen Autoren herausgestellte a-typische Verlauf der Krise nach dem »Absterben des Franquismus (Pries 1987) machte die regionalen und sozialen Disparitäten, die sich unter der »Liberalisierung« vertieft hatten, deutlich.

Der Regulationsansatz wäre durchaus hilfreich, wenn die ungelösten Fragen des Lohnverhältnisses, welche die marxistische Theorie hinterläßt und auf die die Regulationisten hingewiesen haben, in intellektuell redlicher Weise angegangen würden. Aglietta, der als Begründer des modernen Regulationsansatzes gilt, scheint diesen Weg zu gehen (vgl. Hübner/Mahnkopf 1988, S. 41f, Cartelier/de Vroey 1988)), indem er in seinen neueren Arbeiten das Lohnverhaltnis als eine dem Produktionsprozeß exogene Beziehung auffaßt. Wellhöner aber benutzt den Regulationsansatz nur in einer Art theoretischer Selbstbezogenheit - zur Aktualisierung einer abgehalfterten Ideologie. Er hat nicht das Interesse, handlungsleitende Perspektiven zu liefern oder strategische Ziele zu formulieren. Sein Aufsatz läßt nur den resignativen Schluß zu, daß in Spanien keine andere Entwicklung möglich war und auch nicht möglich sein wird. Das macht zwar Stimmung, bietet aber keine Nutzanwendung.

Die bisherige Entwicklung Spaniens, die weitgehend durch partielle oder totale Isolation gekennzeichnet war, wurde bedingt durch die divergierenden, temporär zusammenfallenden Interessen und strategischen Präferenzen der kollektiven Akteure. Daß soziale und regionale Disparităten in Spanien weiterbestehen, ist Ausdruck und Folge des Verhaltens dieser Akteure. Langfristig lassen sich diese Folgen m.E. nur durch die weitere europäische Integration auszugleichen. Gänzlich offen ist jedoch, ob die integrativen Tendenzen in Europa ausreichen werden, um die jüngsten Entwicklungen in Osteuropa und deren wirtschaftliche und soziale Folgen zu kompensieren, oder ob gruppenspezifische, regionale oder nationale Interessen - mit welchem ideologischen Anstrich auch immer - dieses Projekt torpedieren. Die Folgen wären katastrophal.

\section{Literatur}

Bernecker, W. L. (1990): Sozialgeschichte Spaniens in 19. und 20. Jahrhundert, Frankfust/M.

Cartelier, J./de Vroey, M. (1988): Der Regulationsansatz - ein neues Paradigma? in: PROKLA 72, 18. Jg., S. 72-92

De la Dehensa, G. (1985): Las empresas multinacionales en España: planteamiento general, in: Economía industrial, N² 244, Multinacionales en España, Ed.: Sría. Gen. Tec. MINER, S. 75-85

Farre Terre, A. (1986): La regulación de la industria de automoción en España y sus relaciones con el desarollo del sector, in: Economía industrial, N²52, Industria del Automóvil en España, Ed.: Sría. Gen. Tec. MINER, S. 27-43

Frey, P.(1988): Spanien und Europa. Die spanischen Intellektuellen und die Europäische Integration, Bonn

Hübner, K./ Mahnkopf, B. (1988): Ecole de la Régulation. Eine kommentierte Literaturstudie, discussion Paper FS II 88-210, Wissenschaftszentrum Berlin

Lipietz, A. (1985): Akkumulation. Krisen und Auswege aus der Krise. Einige methodische Uberlegun- 
gen zum Begriff der Regulation, in: PROKLA 58, 15. Jg., S. 109-137

Malo de Molina, J. L. (1982): La estructura salarial española por ramas de actividad durante el periodo 1963-1975, in: El mercado de trabajo en España, Ed.: Sría. Gen. Tec, MEC Madrid

Mata, M. (1979): Wachstum und Strukturwandel in der Industrie und im Außenhandel Spaniens, Diss. Hamburg

Ochoa-Westenenk, R. (1986): Industrialisierung und Strukturksise: Zur Entwicklungsökonomie Spaniens, Saarbrücken

Olson, M. (1985): Aufstieg und Fall von Nationen. Ökonomisches Wachstum, Stagflation und soziale Startheit, Tübingen

Pries, L. (1987): Qualifikation, Arbeitsbeziehungen und Arbeitsmarks - die Enge betrieblicher Räume in Spanien, (hrsg. vorn Landesinstitut Sozialforschungsstelle) Dortmund

Serrano Sanz, J. M. (1988): Un siglo de política comercial española en perspectiva, in: Velarde/García/Delgado/Pedreno: El sector exderior de la economía española, Ed.: Economistas, Madrid

Serrano/Malo de Molina (1979): Salarias y mercado de trabajo en España, Madrid

Tamames, R. (1983): La República. La Era de Franco, 10. Aufl. 1983, Alianza Ed. Alfaguara VII, Madrid. 